\title{
Looking at the distant universe with the MeerKAT Array (LADUMA)
}

\author{
B. W. Holwerda ${ }^{1}$, S.-L. Blyth ${ }^{2}$, A. J. Baker ${ }^{3}$ and the LADUMA team \\ ${ }^{1}$ European Space Agency (ESTEC), Keplerlaan 1, 2200 AV Noordwijk, The Netherlands \\ ${ }^{2}$ ACGC, Department of Astronomy, University of Cape Town, South Africa \\ ${ }^{3}$ Rutgers University, USA \\ email: benne.holwerda@esa.int
}

\begin{abstract}
The MeerKAT (64 x 13.5m dish radio interferometer) is South Africa's precursor instrument for the Square Kilometre Array (SKA), exploring dish design, instrumentation, and the characteristics of a Karoo desert site and is projected to be on sky in 2016. One of two top-priority, Key Projects is a single deep field, integrating for 5000 hours total with the aim to detect neutral atomic hydrogen through its $21 \mathrm{~cm}$ line emission out to redshift unity and beyond. This first truly deep HI survey will help constrain fueling models for galaxy assembly and evolution. It will measure the evolution of the cosmic neutral gas density and its distribution over galaxies over cosmic time, explore evolution of the gas in galaxies, measure the TullyFisher relation, measure $\mathrm{OH}$ maser counts, and address many more topics. Here we present the observing strategy and envisaged science case for this unique deep field, which encompasses the Chandra Deep Field-South (and the footprints of GOODS, GEMS and several other surveys) to produce a singular legacy multi-wavelength data-set.
\end{abstract}

Keywords. surveys, galaxies: evolution galaxies: high-redshift galaxies: ISM galaxies: luminosity function, mass function galaxies: statistics cosmology: observations radio lines: galaxies

\section{Introduction}

Over the past $8 \mathrm{Gyr}$, galaxies have evolved dramatically, with the number density of luminous, blue, star-forming galaxies dropping by an order of magnitude (e.g., Bell et al. 2005) and the number of luminous red galaxies increasing by a similar amount (Driver et al. 1998; Bell et al. 2005). Similarly since $z \sim 1$, the star formation rate density in the Universe has plummeted by an order of magnitude (e.g., Madau et al. 1998; Hopkins et al. 2008), yet the density of neutral hydrogen (HI), the fuel reservoir for star-formation, may have remained constant (Fig. 1). Given that, locally, the overall HI mass and starformation rate appear to be linked for galaxies (Kennicutt 1998), this points to significant redistribution of the gas reservoir over this epoch.

To complement our view of high redshift galaxy populations (the stellar, ionised, and molecular gas), a key science goal of the Square Kilometre Array (SKA Carilli \& Rawlings 2004) is to explore the atomic hydrogen (HI) out to $z \sim 1$ and to explore how galaxies form and evolve. While SKA will study HI in large numbers of galaxies at $z \sim 1$, MeerKAT (Booth et al. 2009; de Blok et al. 2009), with its high sensitivity and wide instantaneous bandwidth, is the choice pathfinder instrument with which to begin the study of HI in galaxies out to redshift unity. We therefore are undertaking the LADUMA $†$ (Looking At the Distant Universe with the MeerKAT Array) survey for a 5000 hour observation of a single field (Holwerda \& Blyth 2010a,b; Holwerda et al. 2011a). This survey in combination with existing and planned complementary multi-wavelength

$\dagger$ Literally, It thunders. in the Zulu language: http://www. ast.uct.ac.za/laduma/Home.html 


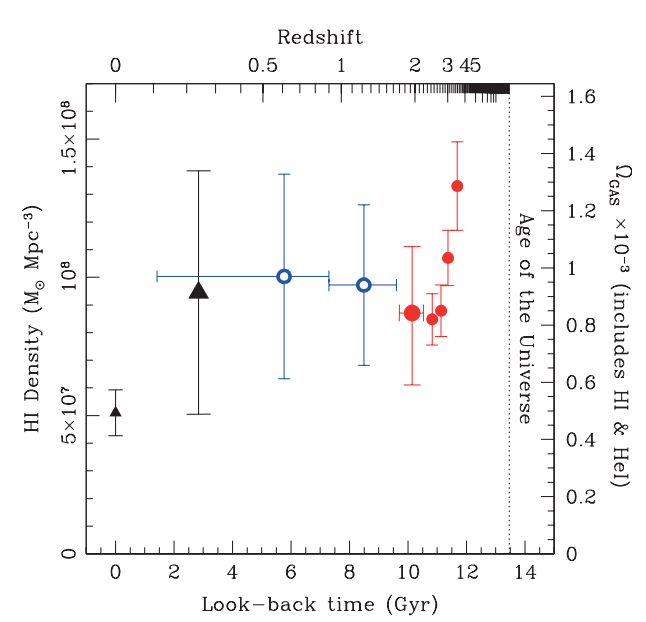

Figure 1. The cosmic HI density from a variety of methods; direct $\mathrm{HI}$ line detections (black tringles Zwaan et al. 2005; Lah et al. 2007), and Ly- $\alpha$ absorption estimates (open and filled circles Rao et al. 2006; Prochaska et al. 2005; Noterdaeme et al. 2009, respectively).

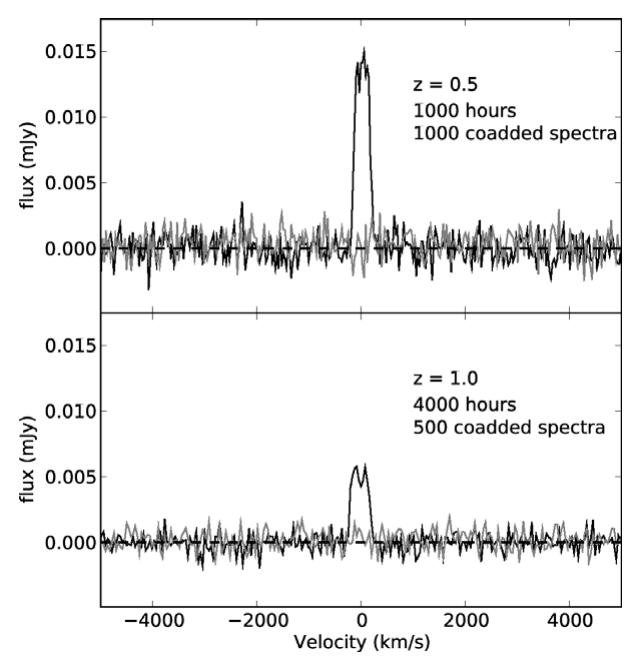

Figure 2. Simulated stacking results (black lines) for $\mathrm{z}=0.5$ (top panel) and $\mathrm{z}=1.0$ (bottom panel $),(\Delta z=0.1)$. Galaxies were simulated according to the Oxford $S^{3}$ database (Obreschkow et al. 2009).

observations will provide the first complete picture of galaxy evolution from $0<z \lesssim 1.4$ and serve as a key benchmark for future studies with the SKA.

\section{Observing Strategy}

Target Field The Extended Chandra Deep Field-South was chosen for its wealth of multiwavelength data and existing spectroscopy (e.g., Cardamone et al. 2010; Balestra et al. 2010) as well as continuous visibility from the MeerKAT site.

Data The final 5000 hour integration results in a trumpet-shaped data-cubet, as the primary beam widens with redshift, covering an ever larger volume with redshift.

Direct Detections \& Line Stacking Given the shape of the survey volume and the gradually decreasing sensitivity with distance, our science will be done with a mix of direct detections (for low redshifts and/or high HI masses) and the stacking of multiple objects with low signal-to-noise (see also Meyer et al. this volume). Fig. 2 illustrates the line stacking technique. Using the known positions and redshifts of a sample of sources, their radio spectra are aligned to a common (rest) frequency frame and combined. One can determine the mean HI mass and possibly the HI profile of a sub-class of galaxies. This technique will require an order of magnitude more spectroscopic redshifts than currently available (Cardamone et al. 2010; Balestra et al. 2010).

\section{LADUMA Science}

The LADUMA data will address a multitude of science questions;

Cosmic Hydrogen Density $\left(\Omega_{H I}\right)$ Summing over all objects, one obtains an estimate of the total atomic hydrogen volume density of the Universe at a given epoch. Given that the

$\dagger$ The vuvuzela is a uniquely South African trumpet primarily used for encouragement during soccer matches, as most of the world knows after the 2010 World Cup. 
star-formation rate density decreases by a factor ten (Hopkins et al. 2008, this volume) and specific star-formation rate by a factor three (Noeske et al. 2007), over the redshift range $0<z \lesssim 1.4$, one can expect the star-formation fuel to be depleted, but at what rate? Our direct HI observations will be compared to those Ly $\alpha$ and $\mathrm{Mg}$ II absorbers (Ménard \& Chelouche 2009; Kanekar et al. 2009).

HI Mass Function (HIMF) is the number density of galaxies as a function of their neu-

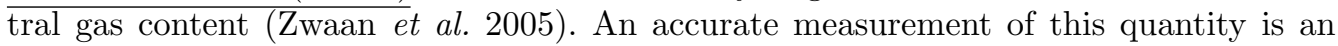
important parameter in models of galaxy evolution where processes like gas inflow and star formation are sought to be understood. Evolution in the breakpoint, slope, and normalization of the HIMF can be used to test hierarchical galaxy formation and cold flow models (van der Heyden et al. 2009). And finally, we can also explore environmental effects on the HIMF (Bouchard et al. 2009).

Galaxy Evolution The HI content of different populations as a function of redshift will place a useful constraint on our understanding of their evolution. We will address questions such as how cold gas mass depends on halo mass? what is the relation between stellar and gas mass over time(e.g., Kannappan 2004), and what is the relation between the cold gas mass and specific star-formation rate?

The Tully-Fisher Relation; Rotationally supported disks lie on the Tully-Fisher relation (Tully \& Fisher 1977). For small high-redshift samples, the stellar T-F relation has been determined (e.g. Kassin et al. 2007; Puech et al. 2010), but the LADUMA data will provide HI data for the first time at higher redshift with the advantage of probing the whole disk (and consequently halo), and with sufficient statistics to determine the normalization and slope of the T-F relation at different redshifts.

Bonus Science includes the number densities and strengths of $\mathrm{OH}$ masers over cosmic time (see Briggs 1998), the merger rate from close companions (e.g., Holwerda et al. 2011b), and the possible detection of faint $21 \mathrm{~cm}$ absorption (e.g., the "Cosmic Web").

Concluding Remarks: The LADUMA survey promises to facilitate a range of exciting science and provide the deepest HI observations available until the SKA is commissioned.

\section{References}

Balestra, I. et al. 2010, A\& A, 512, A12+

Bell, E. F. et al. 2005, ApJ, 625, 23

Booth, R. S. et al. 2009, ArXiv e-prints/0910.2935

Bouchard, A. et al. 2009, in Panoramic Radio Astronomy: Wide-field 1-2 GHz Research on Galaxy Evolution

Briggs, F. H. 1998, A\&A, 336, 815

Cardamone, C. N. et al. 2010, ApJS, 189, 270

Carilli, C. L. \& Rawlings, S. 2004, New A Rev., 48, 979

de Blok, W. J. G. et al. 2009, in Panoramic Radio Astronomy: Wide-field 1-2 GHz Research on Galaxy Evolution

Driver, S. P. et al. 1998, ApJ, 496, L93+

Holwerda, B. \& Blyth, S. 2010a, in ISKAF2010 Science Meeting

Holwerda, B. W. \& Blyth, S. 2010b, ArXiv e-prints/1007.4101

Holwerda, B.W. et al. 2011a, in Bulletin of the AAS, Vol. 43, Abstract \#217, \#433.17

Holwerda, B. W. et al. 2011b, ArXiv e-prints

Hopkins, P. F. et al. 2008, ApJ, 679, 156

Kanekar, N. et al. 2009, MNRAS, 396, 385

Kannappan, S. J. 2004, ApJ, 611, L89

Kassin, S. A. et al. 2007, ApJ, 660, L35

Kennicutt, Jr., R. C. 1998, ApJ, 498, 541 
Lah, P. et al. 2007, MNRAS, 376, 1357

Madau, P. et al. 1998, ApJ, 498, 106

Ménard, B. \& Chelouche, D. 2009, MNRAS, 393, 808

Noeske, K. G. et al. 2007, ApJ, 660, L47

Noterdaeme, P. et al. 2009, A\&A A, 505, 1087

Obreschkow, D. et al. 2009, ApJ, 703, 1890

Prochaska, J. X. et al. 2005, ApJ, 635, 123

Puech, M. et al. 2010, A\&A, 510, A68+

Rao, S. M. et al. 2006, ApJ, 636, 610

Tully, R. B. \& Fisher, J. R. 1977, A\&A, 54, 661

van der Heyden, K. et al. 2009, in Panoramic Radio Astronomy: Wide-field 1-2 GHz Research on Galaxy Evolution

Zwaan, M. A. et al. 2005, MNRAS, 359, L30 